\title{
Chapter 86 \\ Report on the Global Environment \\ Competitiveness of Luxembourg
}

Luxembourg is a landlocked country in western Europe. It covers 2,590 square kilometres and borders by Belgium, France, and Germany. Luxembourg has an oceanic climate, marked by high precipitation, particularly in late summer. It had a population of 0.52 million and domestic production the gross (GDP) of USD 59.2 billion

\begin{tabular}{|l|}
\hline Score: \\
51.66 \\
\hline Rank: \\
48 \\
\hline
\end{tabular}
in 2011. Through the evaluation of global environment competitiveness, we can know that the environment competitiveness index of Luxembourg ranks at 48 among 133 countries.

Fig. 86.1 Contribution of

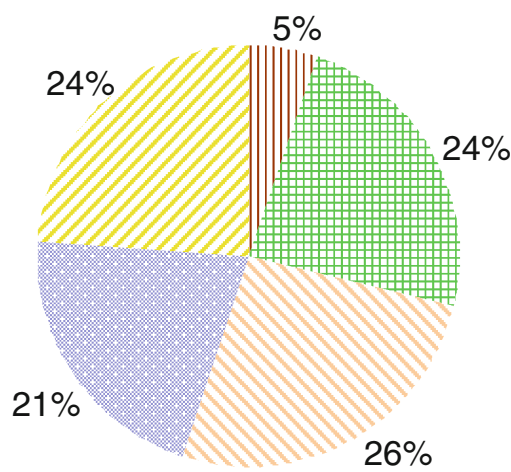

III Resource Environment Competitiveness

\# Ecological Environment Competitiveness Environment Carrying Competitiveness

* Environment Management Competitiveness

Environment Harmony sub-index of GEC

Competitiveness 


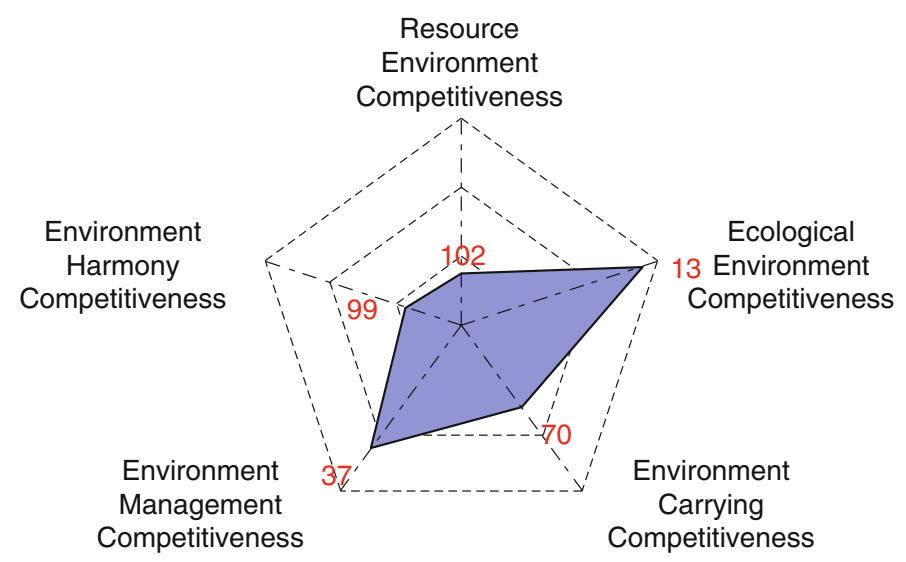

Fig. 86.2 Rank of sub-index of GEC

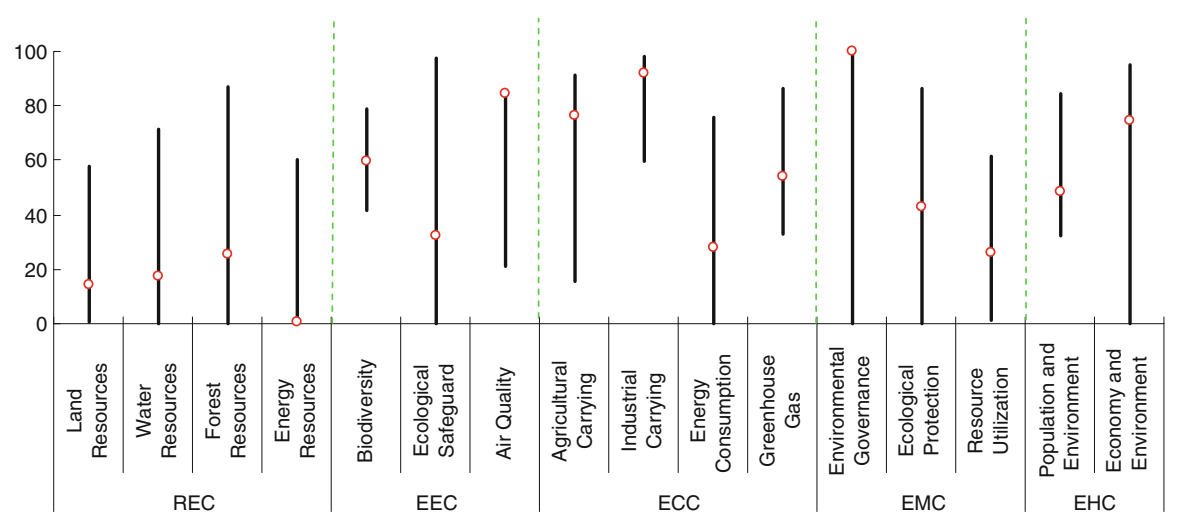

Fig. 86.3 Score and rank of the pillars of GEC

Table 86.1 Score and rank of all indicators of GEC

\begin{tabular}{lrrllrr}
\hline Indicators & Score & Rank & Indicators & Score & Rank \\
\hline $\begin{array}{l}\text { 1 Resource Environment } \\
\quad \text { Competitiveness }\end{array}$ & 13.37 & 102 & Groundwater & 4.41 & 90 \\
1.1 Land Resources & 14.17 & 57 & $\begin{array}{l}\text { Total internal renewable } \\
\text { water resources }\end{array}$ & 19.47 & 53 \\
Land area per capita & 0.88 & 111 & 1.3 Forest Resources & 25.62 & 81 \\
$\begin{array}{l}\text { Percentage of arable land } \\
\quad \text { to total land area }\end{array}$ & 40.47 & 31 & $\begin{array}{c}\text { Growing stock in forest } \\
\text { and other wooded land }\end{array}$ & 50.08 & 102 \\
$\begin{array}{l}\text { Arable land per capita } \\
\text { 1.2 Water Resources }\end{array}$ & 5.59 & 90 & Proportion of land area & N/A & N/A \\
Surface water & 17.12 & 59 & covered by forest & & \\
Annual precipitation & 12.92 & 24 & Forest area per capita & 1.17 & 79 \\
\hline & 31.67 & 63 & 1.4 Energy Resources & 0.91 & 116 \\
\hline
\end{tabular}


Table 86.1 (continued)

\begin{tabular}{|c|c|c|c|c|c|}
\hline Indicators & Score & Rank & Indicators & Score & Rank \\
\hline Fossil energy & 0.00 & 64 & 3.3 Energy Consumption & 27.82 & 124 \\
\hline Energy production & 0.26 & 108 & Energy consumption per unit & 96.52 & 120 \\
\hline \multirow{2}{*}{$\begin{array}{l}\text { Proportion of combustible } \\
\text { renewables and waste to } \\
\text { total energy consumption }\end{array}$} & \multirow[t]{2}{*}{3.92} & \multirow[t]{2}{*}{91} & of land area & & \\
\hline & & & $\begin{array}{l}\text { Ratio of clean energy } \\
\text { consumption }\end{array}$ & 0.72 & 101 \\
\hline $\begin{array}{l}\text { Net energy imports of the } \\
\text { energy consumption }\end{array}$ & 0.24 & 125 & $\begin{array}{l}\text { Elasticity of energy } \\
\text { consumption }\end{array}$ & 12.58 & 115 \\
\hline $\begin{array}{l}2 \text { Ecological Environment } \\
\text { Competitiveness }\end{array}$ & 61.60 & 13 & $\begin{array}{l}\text { Elasticity of electric power } \\
\text { consumption }\end{array}$ & 1.46 & 122 \\
\hline 2.1 Biodiversity & 59.91 & 18 & 3.4 Greenhouse Gas & 54.30 & 116 \\
\hline Threatened fish species & 99.53 & 3 & \multirow{2}{*}{$\begin{array}{l}\text { Growth rate of } \mathrm{CO}_{2} \\
\text { emissions }\end{array}$} & \multirow[t]{2}{*}{46.90} & \multirow[t]{2}{*}{82} \\
\hline Threatened mammal species & 100.00 & 1 & & & \\
\hline Threatened plant species & 100.00 & 1 & Growth rate of Methane & 50.06 & 112 \\
\hline \multirow{2}{*}{$\begin{array}{l}\text { GEF benefits index for } \\
\text { biodiversity }\end{array}$} & \multirow[t]{2}{*}{0.00} & \multirow[t]{2}{*}{128} & ns & \multirow{3}{*}{95.44} & \multirow{3}{*}{127} \\
\hline & & & $\mathrm{CO}_{2}$ emissions pe & & \\
\hline 2.2 Ecological Safeguard & 32.45 & 38 & of land area & & \\
\hline Terrestrial protected areas & 54.08 & 28 & $\mathrm{CO}_{2}$ emissions per unit & \multirow[t]{2}{*}{32.18} & \multirow[t]{2}{*}{97} \\
\hline Marine protected areas & N/A & N/A & of energy consumption & & \\
\hline 2.3 Air Quality & 84.73 & 1 & 4 Environment Management & \multirow[t]{2}{*}{55.05} & \multirow[t]{2}{*}{37} \\
\hline Inhalable particles (PM10) & 91.24 & 9 & Competitiveness & & \\
\hline Particulate matter (PM2.5) & 100.00 & 1 & 4.1 Environmental & \multirow[t]{2}{*}{100.00} & \multirow[t]{2}{*}{1} \\
\hline Index of indoor air pollution & 100.00 & 1 & \multirow{2}{*}{$\begin{array}{l}\text { Governance } \\
\text { Agricultural chemicals } \\
\text { regulation }\end{array}$} & & \\
\hline Nitrogen oxides emission & 68.92 & 8 & & N/A & N/A \\
\hline Sulfur dioxide emission & 40.99 & 5 & \multirow[b]{2}{*}{$\begin{array}{l}\text { Percentage of the rural } \\
\text { population with access to } \\
\text { an improved water source }\end{array}$} & \multirow{2}{*}{100.00} & \multirow{2}{*}{1} \\
\hline $\begin{array}{l}3 \text { Environment Carrying } \\
\text { Competitiveness }\end{array}$ & 66.88 & 70 & & & \\
\hline 3.1 Agricultural Carrying & 76.25 & 23 & \multirow{2}{*}{$\begin{array}{l}\text { Percentage of the urban } \\
\text { population with access to } \\
\text { an improved water source }\end{array}$} & \multirow[t]{2}{*}{100.00} & \multirow{2}{*}{1} \\
\hline $\begin{array}{l}\text { Cereal yield per unit of arable } \\
\text { land }\end{array}$ & 58.94 & 18 & & & \\
\hline $\begin{array}{l}\text { Fertilizer consumption per } \\
\text { unit of arable land }\end{array}$ & 75.59 & 117 & 4.2 Ecological Protection & & 29 \\
\hline $\begin{array}{l}\text { unit of arable land } \\
\text { Annual freshwater }\end{array}$ & 100.00 & 1 & $\begin{array}{l}\text { Area of plantation and } \\
\text { afforestation }\end{array}$ & 0.04 & 102 \\
\hline $\begin{array}{l}\text { withdrawals for } \\
\text { agriculture per unit }\end{array}$ & & & Biome protect & 100.00 & 1 \\
\hline $\begin{array}{l}\text { agriculture per unit } \\
\text { of arable land }\end{array}$ & & & $\begin{array}{l}\text { Overfishing of fishing } \\
\text { resources }\end{array}$ & N/A & N/A \\
\hline 3.2 Industrial Carrying & 91.94 & 60 & 4.3 Resource Utilization & 26.32 & 112 \\
\hline $\begin{array}{l}\text { Net exports as a percentage } \\
\text { of GDP }\end{array}$ & 82.46 & 58 & $\begin{array}{l}\text { Utilization rate of water } \\
\text { resources }\end{array}$ & 0.07 & 95 \\
\hline $\begin{array}{l}\text { Electric power consumption } \\
\text { per unit of value added } \\
\text { of industry }\end{array}$ & 85.82 & 101 & $\begin{array}{l}\text { Percentage of total internal } \\
\text { renewable water resources } \\
\text { to total water resources }\end{array}$ & 31.45 & 107 \\
\hline $\begin{array}{l}\mathrm{SO}_{2} \text { emissions per unit of } \\
\text { value added of industry }\end{array}$ & 99.98 & 16 & $\begin{array}{l}\text { Percentage of agricultural } \\
\text { land to total land area }\end{array}$ & 59.78 & 50 \\
\hline $\begin{array}{l}\text { Annual freshwater } \\
\text { withdrawals for industry } \\
\text { per value added of } \\
\text { industry }\end{array}$ & 99.50 & 20 & $\begin{array}{l}\text { Percentage of fossil fuel } \\
\text { energy consumption to } \\
\text { total energy consumption }\end{array}$ & 13.99 & 85 \\
\hline
\end{tabular}


Table 86.1 (continued)

\begin{tabular}{|c|c|c|c|c|c|}
\hline Indicators & Score & Rank & Indicators & Score & Rank \\
\hline $\begin{array}{l}5 \text { Environment Harmony } \\
\text { Competitiveness }\end{array}$ & 61.39 & 99 & $\begin{array}{l}\mathrm{CO}_{2} \text { emissions (metric tons } \\
\text { per capita) }\end{array}$ & 43.20 & 130 \\
\hline $\begin{array}{l}\text { 5.1 Population and } \\
\text { Environment }\end{array}$ & 48.18 & 129 & $\begin{array}{l}\text { Energy consumption per } \\
\quad \text { capita }\end{array}$ & 35.21 & 124 \\
\hline $\begin{array}{l}\text { Improved sanitation facilities } \\
\text { (\% of population with }\end{array}$ & 100.00 & 1 & $\begin{array}{l}\text { 5.2 Economy and } \\
\text { Environment }\end{array}$ & 74.60 & 3 \\
\hline $\begin{array}{l}\text { access) } \\
\text { Motor vehicles (per 1,000 }\end{array}$ & 7.78 & 132 & $\begin{array}{l}\text { Land resource utilization } \\
\text { efficiency }\end{array}$ & 6.67 & 3 \\
\hline $\begin{array}{l}\text { people) } \\
\text { Renewable internal }\end{array}$ & 2.33 & 82 & $\begin{array}{l}\text { Sulfur dioxide emissions } \\
\text { per unit of GDP }\end{array}$ & 99.85 & 6 \\
\hline $\begin{array}{l}\text { freshwater resources per } \\
\text { capita }\end{array}$ & & & $\begin{array}{l}\text { Carbon dioxide emissions } \\
\text { per unit of GDP }\end{array}$ & 94.55 & 21 \\
\hline $\begin{array}{l}\mathrm{SO}_{2} \text { emissions (metric tons } \\
\text { per capita) }\end{array}$ & 96.74 & 57 & $\begin{array}{l}\text { Energy consumption per } \\
\text { unit of GDP }\end{array}$ & 97.34 & 5 \\
\hline
\end{tabular}

Table 86.2 Rank distribution of the individual indicators of GEC

\begin{tabular}{lllllll}
\hline & $\begin{array}{l}\text { Number } \\
\text { of the } \\
\text { individual } \\
\text { indicators }\end{array}$ & $\begin{array}{l}\text { Rank } \\
1-10\end{array}$ & $\begin{array}{l}\text { Rank } \\
11-30\end{array}$ & $\begin{array}{l}\text { Rank } \\
31-60\end{array}$ & $\begin{array}{l}\text { Rank } \\
61-100\end{array}$ & $\begin{array}{l}\text { Rank } \\
101-133\end{array}$ \\
\hline Sub-index & 14 & 0 & 1 & 3 & 5 & 4 \\
\hline $\begin{array}{l}\text { Resource Environment } \\
\quad \text { Competitiveness } \\
\text { Ecological Environment } \\
\quad \text { Competitiveness }\end{array}$ & 11 & 8 & 2 & 1 & 0 & 0 \\
$\begin{array}{c}\text { Environment Carrying } \\
\quad \text { Competitiveness }\end{array}$ & 15 & 0 & 3 & 2 & 1 & 9 \\
$\begin{array}{c}\text { Environment Management } \\
\quad \text { Competitiveness }\end{array}$ & 10 & 3 & 1 & 1 & 1 & 3 \\
$\begin{array}{c}\text { Environment Harmony } \\
\text { Competitiveness }\end{array}$ & 10 & 4 & 1 & 1 & 1 & 3 \\
Total & 60 & 15 & 8 & 8 & 8 & 19 \\
\hline
\end{tabular}

Open Access This chapter is distributed under the terms of the Creative Commons Attribution Noncommercial License, which permits any noncommercial use, distribution, and reproduction in any medium, provided the original author(s) and source are credited. 